\title{
Walking-posture Classification from Single-acceleration- sensor Data using Deep Learning and its Evaluation
}

\author{
Tessai Hayama *, Rena Arakawa *
}

\begin{abstract}
We described a walking-posture classification method from a single accelerator attached to a human waist using a deep learning technique. We considered deep learning architectures for a single accelerator based on previous human activity recognition studies and investigated the classification accuracy of the proposed method using the walking-posture dataset. The results demonstrate that a deep learning approach to walking-posture classification using a single accelerator is more useful than the conventional SVM approach. Additionally, we also confirmed that a hybrid network architecture with three convolutional neural layers, two pooling layers between the convolutional layers, and a long short-term memory layer achieved the best accuracy of 0.9803 compared to other network architectures. We also confirmed the deep learning approach yielded more correct classification with less periods of each sample for each walking-posture category in spite of the difficulty to detect the classification by the SVM approach.
\end{abstract}

Keywords: Walking-posture classification, Human Activity Recognition, Deep Learning, Health-care

\section{Introduction}

Many researchers have investigated human activity recognition (HAR) based on wearable devices to support health-care or smart home. Particularly, using an accelerator has become very popular in the HAR domain[17, 9]. This study falls in the HAR domain. Here the goal is to detect improper walking postures while walking as a health support activity using a single accelerator.

In HAR technologies based on gait analysis, accelerators are commonly attached to a human waist to recognize general human activities or for human authentication. To diagnose health problems, typically, two accelerator sensors are attached to both sides of a human body. For example, Gadalate et al.[4] developed a human authentication method that analyzed human gait from acceleration data captured using smartphone. Their authentication method combined a machine learning technique, i.e., a Support Vector Machine (SVM), and deep learning. Ordonez et al.[12] developed a machine learning-based method

\footnotetext{
* Nagaoka University of Technology, Niigata, Japan
} 
to classify daily-life activities, such as "walk" and "sit-down," using an accelerator attached to a human waist. In the health-care support domain, Hashiguchi et al.[7] adapted an SVM technique to classify walking postures using acceleration data captured via devices attached to both human ankles. Harmmerla et al.[6] developed a method to diagnose Parkinson's disease that adapted a deep learning technique using acceleration data captured using devices attached to both wrists. Lofti et al.[11] developed a back pain diagnosis system that classifies acceleration data captured at both ankles using a deep learning technique. Chan et al.[2] compared some machine learning methods to determine if a user is a back pain patient acceleration data captured on both feet. Therefore, it is evident that two accelerators attached bilaterally are required to capture human gait features to achieve a health-care system. For daily use of a single accelerator device, the device should be worn on the upper half of the body (i.e., from the waist up). However, using an accelerate to detect the features of both sides of the human body for each walking posture is difficult.

To recognize human activities, most previous HAR studies have adopted machine learning techniques. Deep learning techniques are particularly popular due to their flexibility, ability to fit complex models, and high accuracy. Although deep learning neural network architecture plays a significant role in classification accuracy, a specific neural network architecture that can obtain the best result for all datasets has not yet been identified[17]. Thus, it is necessary to consider neural network architecture for a targeted dataset.

In this paper, we described a walking-posture classification method that uses a single accelerator attached to the human waist using a deep learning technique. In this study, we considered the deep learning network architecture for acceleration data based on previous studies on sequential data analysis in the HAR domain. We conducted an experiment using a walking-posture dataset that included acceleration data for four types of walking postures. The results demonstrate that a deep learning approach to classify walking postures is more useful with even less periods of each sample than the conventional SVM approach, and that hybrid neural network architecture comprising three Convolutional Neural Network layers, two pooling layers between the convolutional layers, and a long short-time memory layer obtained the best accuracy (0.9803) among the other network architectures.

\section{Approach}

HAR technologies that use accelerators classify time-series accelerator data into four categories using a modeling action classifier. In this section, we describe an accelerator and its wearing position. Then, we identify walking postures to consider in this study and the implementation of a deep learning classifier.

\subsection{Accelerator and its wearing position}

We use a single small nine-axis motion sensor attached to a belt near the waist on the user's back, as shown in Figure 1. The sensor runs at 116 frame per second and detects values of a three-axis accelerator, a three-axis gyro sensor, and a three-axis magnetic sensor. Thus, time-series data generated from nine types of sensor values are used as input to a walkingposture classifier. 


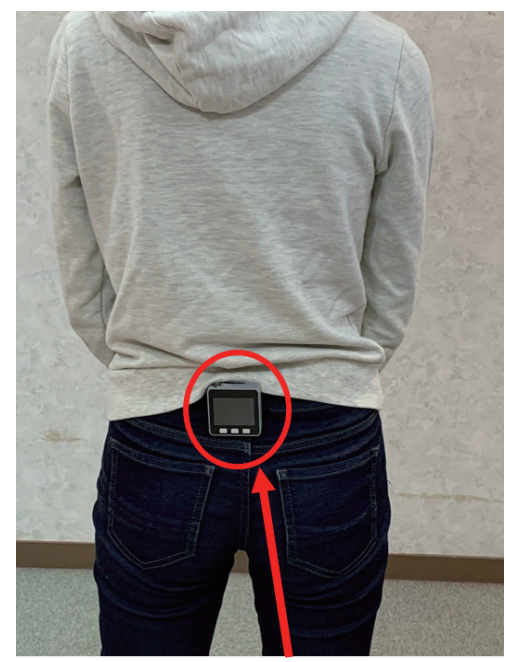

Figure 1: Nine-axis motion sensor and its wearing position.

\subsection{Walking-postures to be classified}

This study is in the beginning stage of walking-posture recognition using a single accelerator. In order to generate a walking-posture dataset, we considered abnormal walking postures from the perspective of physiotherapy[10] and selected walking postures that can be easily simulated.

Abnormal walking postures are generally caused by unbalanced muscle strength and skeleton at the ankle, knee, or hip, and bad walking habits. For example, weak lower limb abductor and abductor muscles often cause "excessive hip adductions," as shown in Figures 2 (a) and (b), respectively. Refraction and inner hip rotations often cause a walking posture with an outward turning heel, as shown in Figure 2(c), which is called "false adduction. " In terms of no cause of muscle weakness, "False adduction" differs from "excessive adduction;" therefore, they can simulate a "false adduction" walking postures.

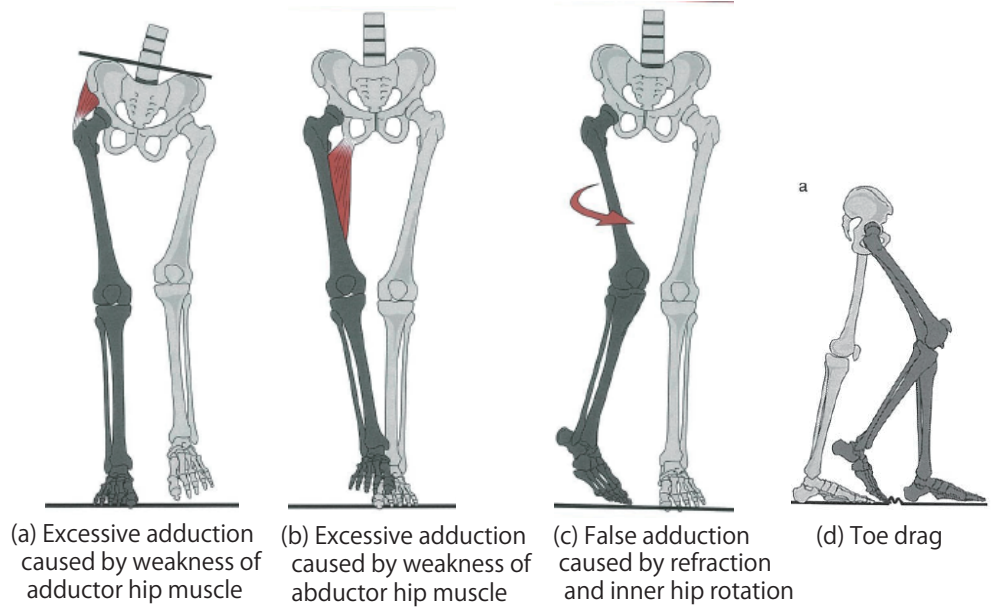

Figure 2: Examples of abnormal walking-posture[10]; adductions and toe drag. 
Therefore, as the beginning stage of walking-posture classification, we selected three types of abnormal walking postures, i.e., "walking posture with toes pointed outward" (as shown in Figure 2(c)), "walking posture with toes pointed inward," and "walking posture with toe drag" (as shown in Figure 2(d)). "Walking posture with toes pointed outward" and "walking posture with toes pointed inward" are simulated by intentionally turning the toes outward and inward at every step, respectively. "Walking posture with toe drag" is simulated by intentionally landing from toe to heel with every step. When using a single accelerator attached to the waist, capturing walking-posture features is difficult because the features of walking-posture motions tend to appear in both lower limbs.

Samples of acceleration data for each walking-postures and the basic statistics of the acceleration data are shown in Figure 3 and Table 1, respectively. Each sensor value in Figure 3 shows 120 frames of time series data from different walking postures of the same person. The basic statistics for each sensor value of the walking postures in Table 1 were computed from the combined time series data of 120 frames collected from six persons.

As shown in Figure 3, although there is periodicity in the time series data of acceleration and angular rate for all walking postures, it is considered difficult to identify clear differences among these walking postures. For example, the Y-axis values for forward/backward acceleration are between 0.6 and -0.4 for all the walking postures. The $\mathrm{Z}$-axis value for up and down acceleration are between 0.4 and -0.6 for the normal walking posture, walking posture with toes pointed outward, and walking posture with toes pointed inward. For all the walking postures, the Y-axis rotation angles stay at about 60 to -40 degrees; for the three walking postures, the Z-axis rotation angles stay at about 80 to -60 degrees. As shown by the basic statistics in Table 1, no statistically significant difference can be confirmed for the different walking postures from the respective means and standard deviations. Thus, highly accurate classification is hardly expected even if the basic statistic is used as a feature of the statistical analysis method, as in the conventional method.

\subsection{Adapting deep learning}

We adapted a deep learning technique for walking-posture classification using a single accelerator attached to the waist. As described in Section 2.2, the walking postures are as follows; "walking posture with toes pointed inward," "walking posture with toes pointed outward," "walking posture with toe drag," and "normal walking posture." In this section, we consider deep learning network architecture based on previous HAR studies using an accelerator.

A Recurrent Neural Network (RNN) is designed to handle sequential data using a deep learning technique. An RNN can adopt long short-term memory (LSTM)[8] architecture, which adds a memory unit and has feedback connections. LSTM architecture enables an RNN to handle sequential data based on long-term dependencies.

Wang et al.[17] surveyed HAR studies that used a deep learning technique and compared the accuracy of some deep learning techniques to those of studies that employed open HAR datasets. The survey results indicated that a Convolutional Neural Network (CNN) and hybrid network architecture comprising a CNN and LSTN could obtain better accuracy for open datasets. Specifically, the CNN[16] with three convolutional layers and pooling layers between the convolutional layers, as shown in Figure 4, obtained the best classification accuracies among some comparison methods using the Skada[13] and UCI smartphone[1] datasets. The DeepConvLSTM[12] having a hybrid network architecture of four convolutional layers and two LSTM layers, as shown in Figure 5, obtained relatively 

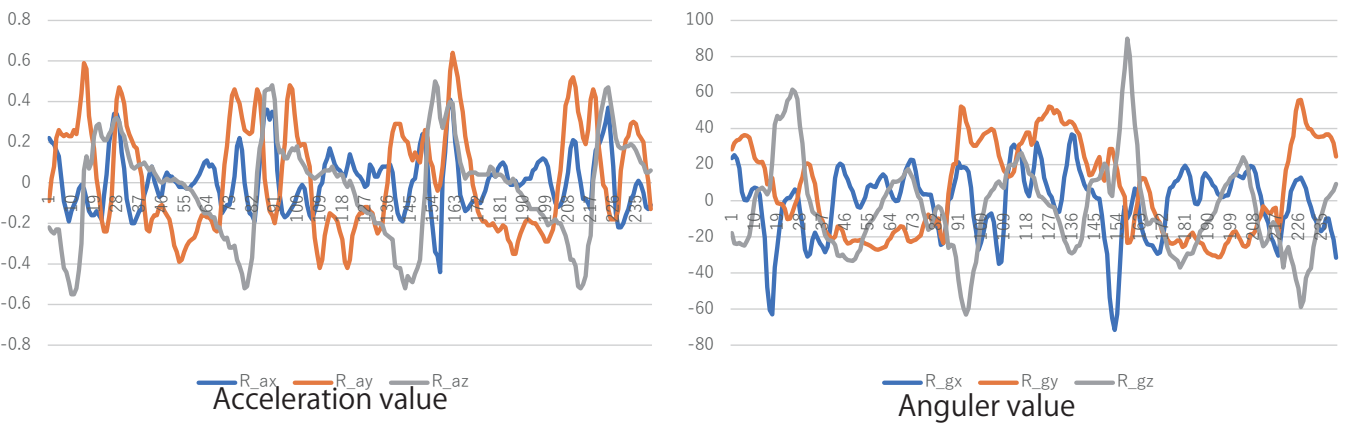

(a) Normal walking-posture
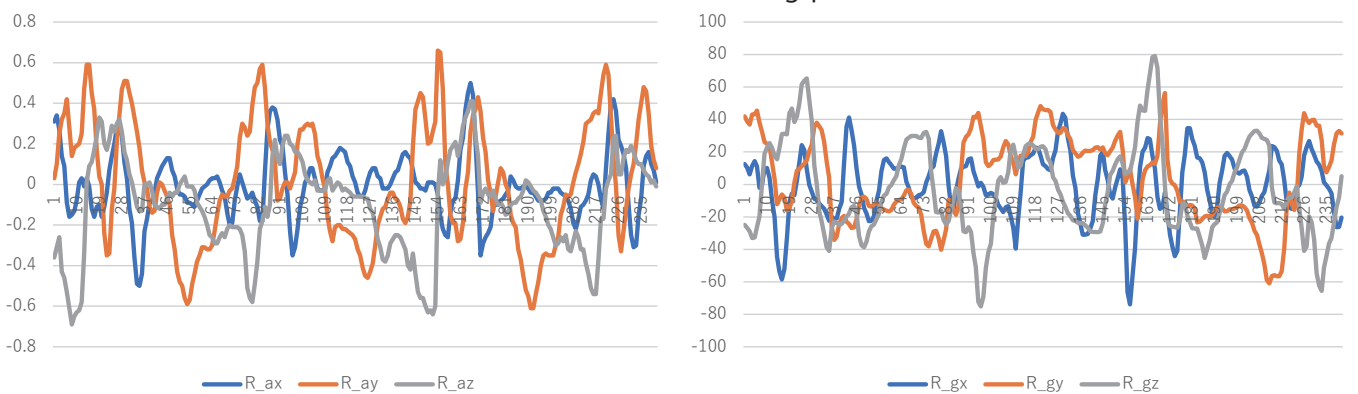

(b) Walking-posture with toes pointed outward
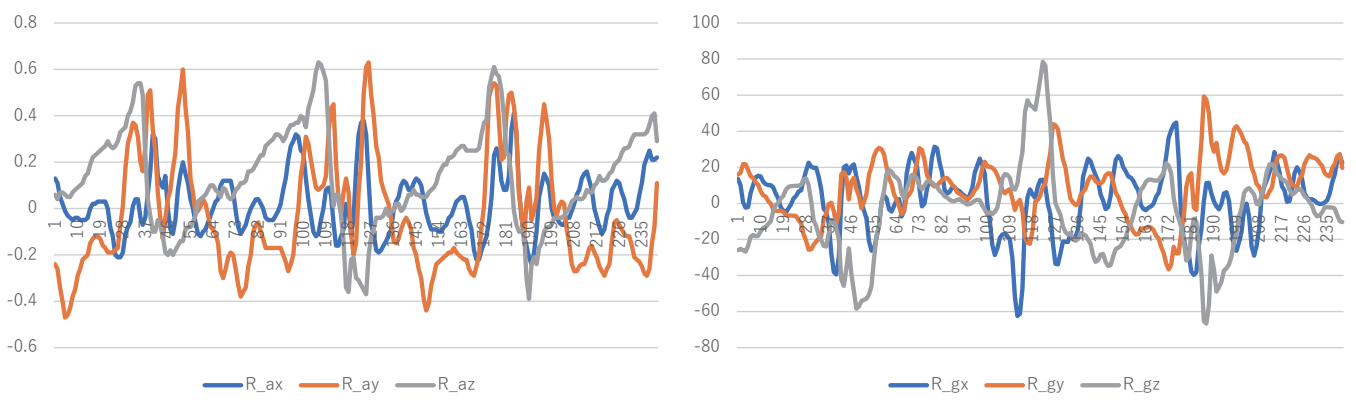

(c) Walking-posture with toes pointed inward
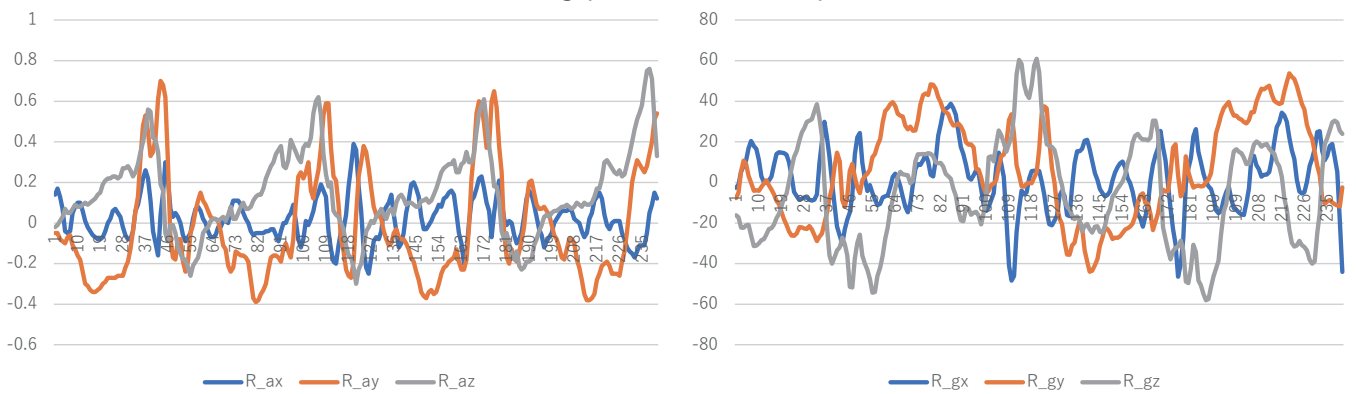

(d) Walking-posture with toe drag

Figure 3: Samples of acceleration value and anguler value for each walking-posture. 
Table 1: Samples of Basic Statistics of acceleration value and anguler value for each walking-posture.

\begin{tabular}{|c|c|c|c|c|c|}
\hline & & Normal & $\begin{array}{c}\text { with toe } \\
\text { pointed outward }\end{array}$ & $\begin{array}{c}\text { with toe } \\
\text { pointed inward }\end{array}$ & $\begin{array}{c}\text { with toes } \\
\text { drag }\end{array}$ \\
\hline \multirow{6}{*}{$\begin{array}{l}\text { Acceleration } \\
\text { value } \\
\text { (X axis) }\end{array}$} & Mean & -0.0028 & 0.0364 & 0.0241 & 0.0209 \\
\hline & Median & -0.0100 & 0.0150 & 0.0100 & 0.0100 \\
\hline & S.D. & 0.2031 & 0.2581 & 0.1987 & 0.1670 \\
\hline & Minimum & -1.2000 & -1.2000 & -0.6500 & -0.8500 \\
\hline & Maximum & 0.7600 & 1.1700 & 0.8700 & 0.7300 \\
\hline & Number of data & 720 & 720 & 720 & 720 \\
\hline \multirow{6}{*}{$\begin{array}{l}\text { Acceleration } \\
\text { value } \\
\text { (Y axis) }\end{array}$} & Mean & 0.0126 & 0.0040 & -0.0158 & -0.0027 \\
\hline & Median & -0.0700 & -0.0700 & -0.0700 & -0.0500 \\
\hline & S.D. & 0.2688 & 0.2891 & 0.2335 & 0.2108 \\
\hline & Minimum & -0.6300 & -0.6100 & -0.4700 & -0.3900 \\
\hline & Maximum & 0.9500 & 1.2500 & 0.9800 & 0.9400 \\
\hline & Number of data & 720 & 720 & 720 & 720 \\
\hline \multirow{6}{*}{$\begin{array}{l}\text { acceleration } \\
\text { value } \\
\text { ( } \mathrm{Z} \text { axis) }\end{array}$} & Mean & 0.0231 & 0.0498 & 0.0618 & 0.0805 \\
\hline & Median & 0.0500 & 0.0900 & 0.0700 & 0.0700 \\
\hline & S.D. & 0.2914 & 0.2852 & 0.2611 & 0.2059 \\
\hline & Minimum & -1.4300 & -1.0000 & -1.3600 & -0.6700 \\
\hline & Maximum & 1.6000 & 0.9100 & 0.9000 & 0.6600 \\
\hline & Number of data & 720 & 720 & 720 & 720 \\
\hline \multirow{6}{*}{$\begin{array}{l}\text { Angular rate } \\
\text { (X axis) }\end{array}$} & Mean & -0.0918 & 0.2315 & 1.8526 & 1.7429 \\
\hline & Median & 2.0150 & 2.4750 & 4.3450 & 2.2100 \\
\hline & S.D. & 36.1121 & 31.6788 & 26.5552 & 20.5261 \\
\hline & Minimum & -181.0800 & -154.8500 & -150.8600 & -111.0200 \\
\hline & Maximum & 232.0300 & 168.6300 & 190.7400 & 59.7500 \\
\hline & Number of data & 720 & 720 & 720 & 720 \\
\hline \multirow{6}{*}{$\begin{array}{l}\text { Angular rate } \\
\text { (Y axis) }\end{array}$} & Mean & 1.1778 & 2.3083 & 1.8945 & 3.1994 \\
\hline & Median & 2.3400 & -3.4850 & 3.8450 & 0.9100 \\
\hline & S.D. & 48.4874 & 48.1673 & 37.3553 & 29.0735 \\
\hline & Minimum & -250.0000 & -194.3200 & -186.5600 & -135.6600 \\
\hline & Maximum & 249.9900 & 237.6900 & 241.3700 & 69.6200 \\
\hline & Number of data & 720 & 720 & 720 & 720 \\
\hline \multirow{6}{*}{$\begin{array}{l}\text { Angular rate } \\
\text { ( } \mathrm{Z} \text { axis) }\end{array}$} & Mean & -3.5375 & -1.8347 & -3.8811 & -4.2440 \\
\hline & Median & -2.6050 & -2.1550 & -4.2650 & -4.3200 \\
\hline & S.D. & 27.1513 & 27.8683 & 22.4293 & 24.6840 \\
\hline & Minimum & -93.4700 & -135.9900 & -82.7900 & -74.6900 \\
\hline & Maximum & 73.6000 & 110.6500 & 79.2700 & 84.9800 \\
\hline & Number of data & 720 & 720 & 720 & 720 \\
\hline
\end{tabular}


good classification accuracy compared to the OPPORTUNITY[12] and Skada[13] datasets. In this papers, the DeepConvLSTM network architecture is referenced as "Conv4LSTM2" to better represent the network architecture.

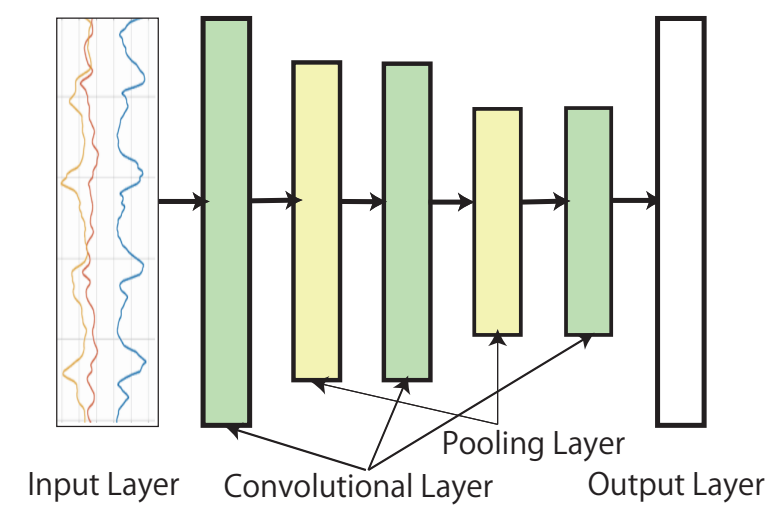

Figure 4: Network architecture of CNN compared in this study.

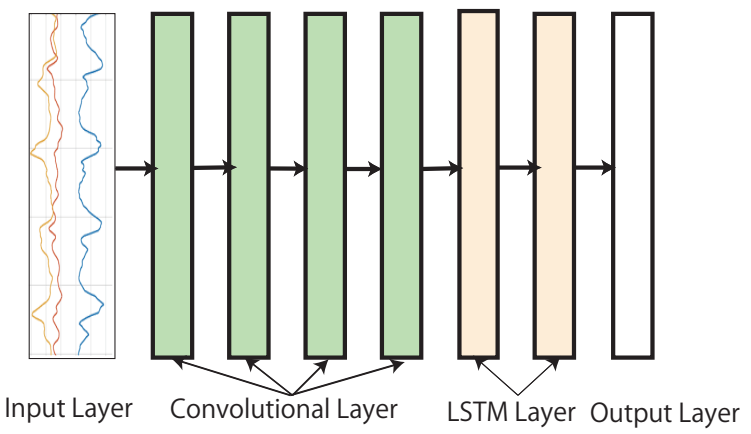

Figure 5: Network architecture of Conv4LSTM2 compared in this study.

DeepSense[15] has been developed as a deep learning algorithm to deal with some types of unified IoT sensor data. The DeepSense algorithm has hybrid network architecture for both of CNN and RNN. The CNN layer contains two types of layers for individual sensors and unification on them. Differing from DeepSense network architecture, in this study, we adopt a hybrid network architecture comprised of a CNN and LSTM architecture, as shown in Figure 6. The first CNN layer is a two-dimensional filter. The second and third layers are one-dimensional filters. There are pooling layers between the filter layers. LSTM is adopted rather than a GNU since LSTM is more general and there is no significant difference between the two architectures[3]. Note, in this paper, the DeepSense network architecture is represented as "Conv3P2LSTM."

Therefore, we compare four types of network architectures (LSTM, CNN, Conv4LSTM2, and Conv3P2LSTM) to adapt deep learning to walking-posture classification. 


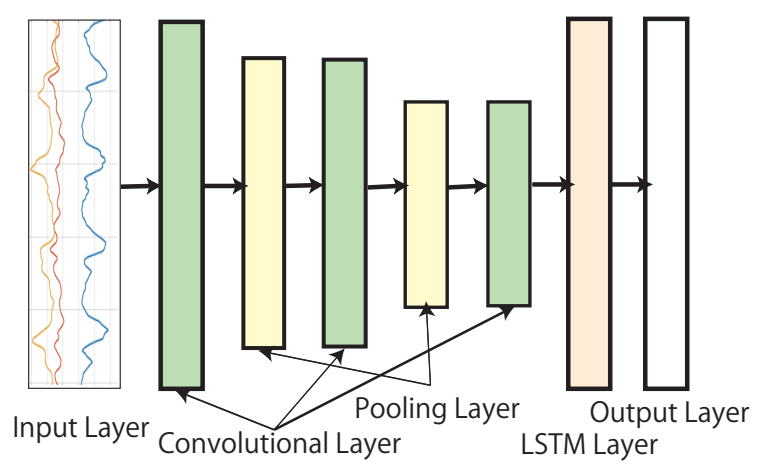

Figure 6: Network architecture of Conv3P2LSTM compared in this study.

\section{Experiment}

\subsection{Overview}

We conducted an experiment using a dataset that contained acceleration data from four types of walking postures to investigate useful deep learning network architecture. We compared the LSTM, CNN, Conv4LSTM2, and Conv3P2LSTM network architecture to two types of SVMs using the features from Principal Component Analysis (PCA) results[7] and features based on Basic Statistics(BS)[5]. For the SVM features from PCA results, 112dimension PCs having a cumulative contribution ratio greater than 0.80 were used to reduce the acceleration data parameters. For the SVM features based on BS, average value, median, standard deviation, skewness, and kurtosis for accelerator and gyro sensor per sample and minimum value, maximum value, and minimum-maximum difference for accelerator per sample were adopted to summarize the features of each sample following a previous study[5].

The programming languages and deep learning frameworks used in this experiment were Python and Tensorflow. The input data for deep learning was 9 (each value of the 9axis accelerometer) $\times 160$ (frames). It is known that the learning effect is degraded when the input data contains bias. Therefore, for each input, the normalized value of the difference between the mean value of all data and each data divided by the standard deviation of the input data was used as the input data. Softmax, Adaptive Moment Estimation, and multi-class cross-entropy were set for the activation function, optimization function, and loss learning of the final layer of deep learning, respectively. A filter size of $5 \times 5$ and a stride of 1 were set for the convolutional layer, and a pooling size of $3 \times 3$, a stride of 1 , and a Max pooling operation were set for the pooling layer. The hidden layer of the LSTM consists of 128 nodes. The SVM in this experiment was implemented in Python using the scikit-lean library, and multi-class classification was performed using the Gaussian kernel and the one-versus-the-rest method.

To create the dataset of walking-posture acceleration data (Section 2.2), we recruited six men in their twenties. We attached a motion sensor to their belts and made them do three round trips in a $25 \mathrm{~m}$ corridor for each participant, motion data from the first two round trips were used for training data and data from the third trip was used for test data. A single unit of training or test data contains nine types of sensor data in 160 frames (i.e., walking for 1.38 seconds). Each data sample was generated with a sliding window length 
of 160 frames. The properties of the generated dataset are shown in Table 2.

Table 2: Properties of the dataset of motion-sensor data for this experiment.

\begin{tabular}{c|cccc|c}
\hline Data & Normal & $\begin{array}{c}\text { with toes } \\
\text { pointed } \\
\text { outward }\end{array}$ & $\begin{array}{c}\text { with toes } \\
\text { pointed } \\
\text { inward }\end{array}$ & $\begin{array}{c}\text { with toe } \\
\text { drag }\end{array}$ & Total \\
\hline Training & 27,161 & 29,655 & 30,400 & 31,810 & 119,026 \\
Test & 13,515 & 14,400 & 15,310 & 16,460 & 59,685 \\
\hline
\end{tabular}

\subsection{Results and discussions}

The experimental walking-posture classification results obtained using deep learning and an SVM are shown in Table 3.

Table 3: Experimental walking-posture classification results obtained using deep learning and an SVM.

\begin{tabular}{l|ccc}
\hline & ACC & ACC+GYRO & ACC+GYRO+COM \\
\hline LSTM & 0.9485 & 0.9731 & 0.9549 \\
CNN & 0.9594 & 0.9771 & 0.9671 \\
Conv4LSTM2 & 0.9302 & 0.9763 & 0.9744 \\
Conv3P2LSTM & 0.9553 & 0.9799 & 0.9803 \\
\hline SVM(PCA) & 0.7076 & 0.8400 & 0.9090 \\
SVM(BS) & \multicolumn{3}{|c}{0.7797}
\end{tabular}

PCA and BS represent principal component analysis and basic statistics, respectively. ACC, GYRO, and COM represent each dataset of accelerator, gyro-sensor, and magnetic-sensor, respectively.

Table 4: Confusion matrix of the walking-posture classification using deep learning having the network architecture of Conv3P2LSTM.

\begin{tabular}{|c|c|c|c|c|}
\hline & Normal & $\begin{array}{l}\text { with toes } \\
\text { pointed outward }\end{array}$ & $\begin{array}{l}\text { with toes } \\
\text { pointed inward }\end{array}$ & $\begin{array}{l}\text { with toe } \\
\text { drag }\end{array}$ \\
\hline \multirow{4}{*}{$\begin{array}{l}\text { Normal } \\
\text { with toes pointed outward } \\
\text { with toes pointed inward } \\
\text { with toe drag }\end{array}$} & 0.9553 & 0.0107 & 0.0160 & 0.0180 \\
\hline & 0.0029 & 0.9923 & 0.0044 & 0.0003 \\
\hline & 0.0400 & 0.0043 & 0.9426 & 0.0131 \\
\hline & 0.0097 & 0.0139 & 0.0106 & 0.9658 \\
\hline
\end{tabular}

The results show that deep learning classification accuracy (greater than 0.97) for the accelerator and gyro sensor dataset of walking postures was better than that obtained by SVM approaches (less than 0.91). Thus, adapting the deep learning technique to walkingposture classification using a single accelerator attached to the waist is useful. In addition, the results also show that the Conv3P2LSTM deep learning network architecture obtained the best classification accuracy (0.9803) among the compared methods. 
Table 5: Confusion matrix of the walking-posture classfication using a SVM.

\begin{tabular}{l|llll}
\hline & Normal & $\begin{array}{l}\text { with toes } \\
\text { pointed outward }\end{array}$ & $\begin{array}{l}\text { with toes } \\
\text { pointed inward }\end{array}$ & $\begin{array}{l}\text { with toe } \\
\text { drag }\end{array}$ \\
\hline Normal & 0.7230 & 0.0330 & 0.1452 & 0.0989 \\
with toes pointed outward & 0.0240 & 0.8463 & 0.0787 & 0.0510 \\
with toes pointed inward & 0.0696 & 0.1231 & 0.5471 & 0.2602 \\
with toe drag & 0.0699 & 0.0225 & 0.0206 & 0.8871 \\
\hline
\end{tabular}

Next, we investigated the classification accuracy for each walking-posture category. The confusion matrices of the walking-posture classification using deep learning and an SVM are shown in Tables 4 and 5, respectively.

As shown in Table 4, the classification method using the deep learning technique achieved accuracies greater than 0.94 in all of walking-posture categories. On the other hand, as shown in Table 5, the SVM classifier often miscategorized "walking posture with toes pointed inward" as either "walking posture with toes pointed outward" and "walking posture with toe drag." The SVM classifier also often miscategorized "normal walking posture" as "walking posture with toes pointed inward." Therefore, the deep learning approach to walking-posture classification can effectively extract the features of each walking posture from a single accelerator attached to the waist, though it is difficult for a single accelerator attached to the human torso to detect both the left and right side of walking motion.

Next, we conducted a logistic regression analysis using the basic statistics of the accelerometer used for SVM walking-posture classification, and then investigated the effective features for walking-posture classification. Table 6 shows the results of the logistic regression analysis of the top-30 features and their weights.

As the results in Table 6 show, the minimum value of acceleration in the $\mathrm{Z}$ direction (longitudinal direction) had the most influence on the classification, with a weight coefficient of 0.0685 . In the next rank, the difference between the maximum and minimum angular rate, the median value of acceleration in the $\mathrm{Z}$ direction had effects with weight coefficient of 0.0428 and 0.0373 , respectively. The minimum value of acceleration and the minimum value of angular rate in the $\mathrm{Y}$ direction had effects with 0.0369 and 0.0366 , respectively. Thus, the influence of the acceleration and angular rate in the $\mathrm{Z}$ and $\mathrm{Y}$ directions occupied the top 12 positions, indicating that the forward and upward movements have a large influence on the walking posture classification.

In addition, we investigated the classification accuracy for periods of each sample. The accuracies of the walking-posture classification for sampling periods of 40, 80, 120, 160, and 200 frames $(0.35 \mathrm{~s}, 0.69 \mathrm{~s}, 1.38 \mathrm{~s}, 1.73 \mathrm{~s}$ and $2.07 \mathrm{~s})$ with using deep learning and an SVM are shown in Figure 7.

In the sampling periods investigated in the experiment, the deep learning methods obtained higher accuracies of the walking-posture classification than the conventional SVM method. In particular, the walking-posture classification with deep learning approach achieves over $90 \%$ accuracy for more than 80 frames per sample $(0.38 \mathrm{~s})$. In previous gait analysis studies, it took five walk-cycles and 30 seconds to detect the walking-posture features using the combined SVM and deep learning method [14] and the Hidden Markov Model method [4], respectively. Therefore, it is found that the deep learning method can capture walking-posture features with less period of each sample than the conventional methods. 
Table 6: Results of Logistic Regression Analysis for Basic Statistics of Accelerometers in Walking Posture Classification.

\begin{tabular}{l|ll}
\hline Rank & Feature & Weight \\
\hline 1 & Min. value of acceleration (Z axis) & 0.0685 \\
2 & Difference between min. and max. angular rate (Z axis) & 0.0428 \\
3 & Median value of acceleration (Z axis) & 0.0373 \\
4 & Min. value of acceleration (Y axis) & 0.0369 \\
5 & Min. value of angular rate (Y axis) & 0.0366 \\
6 & Mean value of acceleration (Z axis) & 0.0363 \\
7 & Kurtosis of acceleration (Y axis) & 0.0357 \\
8 & Max. angular rate (Z axis) & 0.0355 \\
9 & S.D. of acceleration (Y axis) & 0.0333 \\
10 & S.D. of acceleration (Z axis) & 0.0328 \\
11 & S.D. of angular rate (Z axis) & 0.0321 \\
12 & Max. value of acceleration (Z axis) & 0.0318 \\
13 & Average value of Acceleration (X axis) & 0.0293 \\
14 & S.D. of acceleration (X axis) & 0.0291 \\
15 & Max. value of Acceleration (X axis) & 0.0264 \\
16 & Max. value of Acceleration (Y axis) & 0.0263 \\
17 & Min. value of acceleration (X axis) & 0.0260 \\
18 & Skewness of Acceleration (Z-axis) & 0.0253 \\
19 & Difference between min. and max. value of acceleration (Z axis) & 0.0252 \\
20 & Difference between min. and max. angular rate (Y axis) & 0.0250 \\
21 & S.D. of angular rate (Y axis) & 0.0241 \\
22 & Max. value of angular rate (X axis) & 0.0225 \\
23 & Difference between min. and max. value of acceleration (Y axis) & 0.0212 \\
24 & Difference between min. and max. value of acceleration (X axis) & 0.0205 \\
25 & Min. value of angular rate (X axis) & 0.0187 \\
26 & S.D. of angular rate (X axis) & 0.0185 \\
27 & Difference between min. and max. angular rate (X axis) & 0.0184 \\
28 & Max. value of angular rate (Y axis) & 0.0184 \\
29 & Min. value of angular rate (Z axis) & 0.0175 \\
30 & Median value of acceleration (X axis) & 0.0172 \\
31 & Kurtosis of angular rate (Z axis) & 0.0147 \\
32 & Skewness of acceleration (Y axis) & 0.0137 \\
33 & Kurtosis of angular rate (Y axis) & 0.0134 \\
34 & Skewness of angular rate (X axis) & 0.0124 \\
35 & Skewness of acceleration (X axis) & 0.0100 \\
\hline & & \\
& &
\end{tabular}




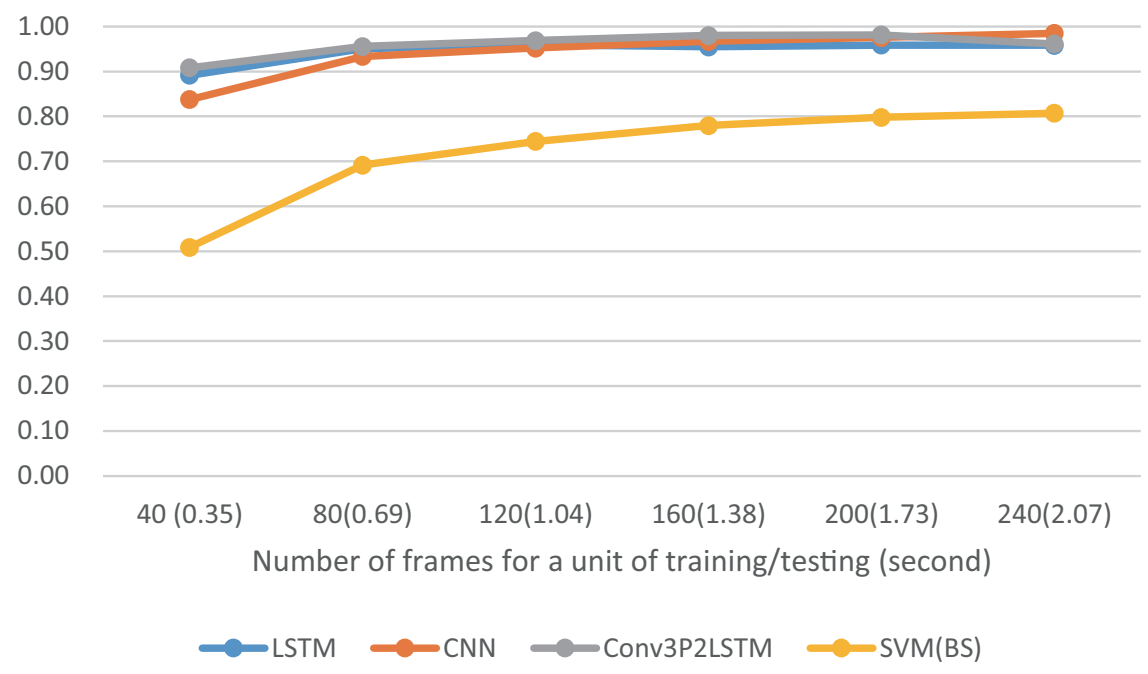

Figure 7: Experimental walking-posture classification results for periods of each sample obtained using deep learning and an SVM.

\section{Conclusion}

In this paper, we described walking-posture classification using deep learning and data from a single accelerator attached to the human waist. The overall objective of the study was to develop a walking-health-care support system for daily use. It is difficult for a single accelerator attached to the waist to capture the walking-posture features because walking motion features tend to appear on both lower limbs. We considered various deep learning network architectures based on previous HAR studies using an accelerator. We conducted an experiment using a walking-posture dataset that included acceleration data of "walking posture with toes pointed inward," "walking posture with toes pointed outward," "walking posture with toe drag," and "normal walking posture." The experimental results demonstrated that the deep learning approach obtained classification accuracy of greater than 0.97 , which was more useful to classify the walking postures with less period of each sample than the conventional methods. In addition, the results also showed that the deep learning technique with hybrid network architecture comprised of three CNN layers, two intermediate pooling layers, and an LSTM layer obtained the best classification accuracy (0.9803) among the compared methods. We confirmed that the deep learning approach can well extract the features of each walking posture from a single accelerator attached to the human waist,

In future, we intend to develop a walking-health-care support system. We need to add additional improper walking postures into the categories adopted in this study and investigate the classification accuracy. Then, we are going to incorporate the walking-posture classification method in daily-human-activity-recognition technology and confirm the usefulness of walking-posture classification in practice. 


\section{Acknowledgment}

This work was supported by JSPS KAKENHI Grant Number 19K12264.

\section{References}

[1] Almaslukh B., AlMuhtadi J., Artoli A. An Effective Deep Autoencoder Approach for Online Smartphone-based Human Activity Recognition. Int. J. Comput. Sci. Netw. Secur. 17:160, 2017.

[2] Chan, H., Zheng, H., Wang, H., Sterritt R., Newell, D. Smart Mobile Phone based Gait Assessment of Patients with Low Back Pain, Procs. 9th International Conference on Natural Computation, pp.1062-1066, 2013.

[3] Chung, J., Caglar G., Cho, K. and Bengio, Y. Empirical Evaluation of Gated Recurrent Neural Networks on Sequence Modeling. ArXiv abs/1412.3555, 2014.

[4] Gadaleta, M., Rossi, M. IDNet: Smartphone-based Gait Recognition with Convolutional Neural Networks, Pattern Recognition, Vol.74, pp.25-37, 2018.

[5] Karvekar, S. B. Smartphone-based Hauman Fatigue Detection in an Industrial Environment Using Gait Analysis. Thesis. Rochester Institute of Technology, 2019.

[6] Hammerla, N. Y., Fisher, J. M., Andras, P., Rochester, L., Walker, R., Plotz., T.PD Disease State Assessment in Naturalistic Environments Using Deep Learning. Procs. the 29th AAAI Conference on Artificial Intelligence. AAAI Press, pp.1742-1748, 2015.

[7] Hashiguchi, H., Hori, K., Hirobe, Y., Sawada, H. Inaba, A., Orimo, S., Miyake, Y. Early Detection System of Parkinson's Disease Based on the Walking Trajectory Data. the 28th SICE Symposium on Decentralized Autonomous Systems, pp.155-158, 2016 (in Japanese).

[8] Hochreiter, S., Schmidhuber, J. Long short-term memory. Neural Computation 9 (8),pp.1735-1780, 1997.

[9] Jordao, A., Nazare Jr, A. C., Sena, J., Schwartz W. R. Human Activity Recognition Based on Wearable Sensor Data: A Standardization of the State-of-the-art, arXiv preprint arXiv:1806.05226, 2018.

[10] Kirsten G. N. Observational Gait Instructor Group, IGAKU-SHOIN Ltd., 2005.

[11] Lotfi, A., Nguyen, M., Langensiepen, C. Human Gait Classification Using a Tri-axial Accelerometer. Procs. the 8th ACM International Conference on PErvasive Technologies Related to Assistive Environments, Article 36, pp.1-4, 2015.

[12] Ordonez, F., Roggen, D. Deep Convolutional and LSTM Recurrent Neural Networks for Multimodal Wearable Activity Recognition. Sensors 16(1), pp.1533-1540, 2016.

[13] Plotz, T., Hammerla, N. Y., Olivier, P. Feature learning for activity recognition in ubiquitous computing. Procs. the 22nd IJCAI, Vol.2. AAAI Press, pp.1729-1734, 2011. 
[14] Nickel, C., Busch, C., Rangarajan, S., Mobius, M. Using Hidden Markov Models for accelerometer-based biometric gait recognition, IEEE 7th International Colloquium on Signal Processing and its Applications, Penang, pp.58-63, 2011.

[15] Yao, S., Hu, S., Zhao, Y., Zhang, A., Abdelzaher, T. Deepsense: A unified Deep Learning framework for time-series mobile sensing data processing. Procs. the 26th International Conference on World Wide Web, pp.351-360, 2017.

[16] Yang, J. B., Nguyen, M. N., San, P. P., Li, X. L., Krishnaswamy, S. Deep Convolutional Neural Networks on Multichannel Time Series for Human Activity Recognition. Procs. the 24th International Conference on Artificial Intelligence. AAAI Press, pp.3995-40010, 2015.

[17] Wang, J., Chen, Y., Hao, S., Peng, X., Hu, L. Deep Learning for Sensor-based Activity Recognition: A Survey. Computer Vision. Pattern Recognition Letters. pp.3-11, 2017. 